British Journal of Psychiatry (1996), 169, 790-796

\title{
Correspondence
}

Contents: Influenza and schizophrenia/The uses and limitations of mental health service needs indices/Treatment of severe personality disorders/ Who responds to electroconvulsive therapy/ Artificial neural networks and psychiatric diagnosis/Lithium and ECT in combination/False memory syndrome/Baclofen therapy for rigidity associated with Lewy body dementia/An atypical case of parasuicide or 'bradysuicide'.

\section{Influenza and schizophrenia}

SIR: Adams \& Kendell (1996) discuss the power of the follow-up study of Cannon et al (1996) to detect maternal influenza during pregnancy as a cause of subsequent schizophrenia in the child. They conclude that the study lacks sufficient power to detect the "significantly increased incidence of schizophrenia in the offspring of women exposed to that [1957] epidemic during the second trimester of pregnancy" which they believe has been demonstrated by their own and others' ecological studies. They conclude "that the size of the cohort [ $n=80$ exposed in the second trimester] was hopelessly inadequate. Similar criticisms apply to an earlier study of similar design by Crow \& Done (1992). Even though they had 945 mothers with a history of flu-like illness in the second trimester of pregnancy they only had 7 schizophrenic offspring."

Adams \& Kendell appear to have made two errors, the first trivial, but the second crucial to the conclusion they wish to reach. First, among the children born to the 945 mothers with influenza-like illnesses in the second trimester, there was one case of schizophrenia by narrow diagnostic criteria (the PSE S + category), and three cases by broad criteria (including categories $\mathrm{S}, \mathrm{P}$ and $\mathrm{O}$ ). The figure of seven can be obtained only by taking the figures for the third rather than the second trimester and adding together the broad and narrow categories (a mistaken aggregation, if it was adopted, since the former includes the latter).

Second, Adams \& Kendell have overlooked the fact that the design of the study differed from that of Cannon et al (1996) in that the 945 mothers with influenza-like illnesses in the second trimester (which coincided with the peak of the 1957 epidemic) were part of a larger population of 16268 mothers who gave birth in the week 3-9 March 1958. For this population not only do we know the numbers of mothers who (according to the information available to the midwife at the time of birth) suffered from an influenza-like illness in the first (231), second (945) and third (675) trimesters, but we also know the number of mothers $(14417)$ who (by these same criteria) did not suffer from an influenza-like illness in pregnancy, and we know how many children ( 57 by broad and 34 by narrow criteria) born to the cohort as a whole later developed schizophrenia.

The key point is this. If we assume, as do Adams \& Kendell (1996), that exposure to influenza in the second trimester increases the risk of schizophrenia (say by a factor of two) we can calculate how many of the births of pre-schizophrenic children in the week 3-9 March 1958 would have been expected to mothers who suffered from influenza-like illnesses in the second trimester and how many would be expected to the rest of the sample. Since Mednick et al (1988) had claimed an $87 \%$ increase in schizophrenic births between 15 February and 14 May 1958 , and O'Callaghan et al (1991) claimed an $88 \%$ increase between 15 February and 14 March 1958 attributable to the 1957 epidemic, we used the figure of 1.87 rather than the figure of 2 suggested by Adams \& Kendell. The implication is that $0.87 / 1.87$ of the total births of pre-schizophrenic children would be to the mothers exposed to influenza. On this basis we calculated that 29.5 cases of schizophrenia by broad and 16.8 cases by narrow criteria would be expected to be born to the 945 mothers. Since the actual numbers were two cases and one case, respectively, we concluded that the hypothesis was not supported.

Since Adams \& Kendell have expressed their conclusions in a forthright manner (a sample of 945 mothers with influenza-like illness in the second trimester was "hopelessly inadequate" and it is "quite wrong ... to suppose that their negative findings, or those of Crow \& Done, weaken that [ecological] evidence") it is perhaps permissible to 
venture that they are themselves mistaken and that the evidence cited above effectively undermines the case that prenatal exposure to influenza is a cause of schizophrenia. I would be more cautious in expressing this opinion were I not also convinced that the ecological evidence that they cite is fraught with inconsistencies and contradictions (Crow, 1994).

ADAMS, W. \& KENDELl, R. E. (1996) Influenza and schizophrenia (letter). British Journal of Psychiatry, 169, 252-253.

Cannon, M., Cotter, D., Cofrey, V. P., et al (1996) Prenatal exposure to the 1957 influenza epidemic and adult schizophrenia: a follow-up study. British Journal of Psychiatry, 168, 368-371.

Crow, T. J. (1994) Prenatal exposure to influenza as a cause of schizophrenia. There are inconsistencies and contradictions in the evidence. British Journal of Psychiatry, 164, 588-592.

Crow, T. J. \& DONE, D. J. (1992) Prenatal exposure to influenza does not cause schizophrenia. British Journal of Psychiatry. 161 390-393.

Mednick, S. A., Machon, R. A., Huttunen, M. O., et al (1988) Adult schizophrenia following prenatal exposure to an influenza epidemic. Archives of General Psychiatry, 45, 189-192.

O’Callaghan, E., Sham, P., TAke, N., et al (1991) Schizophrenia after prenatal exposure to the 1957 A2 influenza epidemic. Lancet, 337, 1248-1250.

\section{Warneford Hospital \\ Headington \\ Oxford OX3 7JX}

T. J. CROW

REPLY: In our recent letter (Adams \& Kendell 1996) about a paper by Cannon et al (1996) we made a passing reference to an earlier study by Crow \& Done (1992). Crow now claims that our argument involved two errors, "the first trivial ... the second crucial".

The first is easily disposed of. It arises out of our statement about Crow \& Done's study that "even though they had 945 mothers with a history of flu-like illness in the second trimester of pregnancy they only had 7 schizophrenic offspring". The disputed figure 7 refers to the total number of offspring with a diagnosis of schizophrenia (broad diagnostic criteria) produced by the 1851 women describing a flu-like illness during pregnancy $(0+3+4)$ in Crow \& Done's Table 2; that is, "they" in the sentence above refers to Crow \& Done, not to the 945 mothers, and we apologise for the ambiguity.

We do not accept, however, that our argument was fallacious. On the contrary, we believe that there is a serious error in Crow \& Done's arithmetic, which Crow repeats in his present letter. With the data available to them Crow \& Done could have used either or both of two arguments to justify their claim to have established that exposure to influenza in the second trimester of pregnancy is not associated with any increased risk of schizophrenia in the child. They could have argued that there was no significantly increased risk of schizophrenia after exposure to influenza in the second compared with the first and third trimesters of pregnancy. The point of our passing reference was that the total number of cases of schizophrenia (7) identified after maternal influenza at any stage of pregnancy was far too small for this to be practicable. Instead, Crow \& Done chose to compare the risk of schizophrenia in the offspring of the 945 mothers describing flu-like illness in the second trimester of pregnancy with the risk in all 16268 mothers in the NCDS cohort, $88.6 \%$ of whom had not described a flu-like illness at any stage of pregnancy, and set out to test the hypothesis that the risk of schizophrenia after maternal influenza in the second trimester of pregnancy was increased by a factor of 1.87 (very similar to our hypothesis that the risk is doubled). They then suggested that this hypothesis required the excess number of schizophrenic births (broad diagnostic criteria) to "be $26.5(57 \times 0.87 / 1.87)$ " and the observed number to be 29.5 rather than 3 .

This is, quite simply, wrong. Using broad diagnostic criteria, 57 schizophrenics were identified by Crow \& Done among the index children of the 16268 mothers in the NCDS cohort. This represents one schizophrenic child for every 285.4 mothers, and one schizophrenic child for every 283.8 mothers among the 15323 who did not report a flu-like illness in the second trimester of pregnancy. This rather low yield is presumably attributable to the imperfect means of case ascertainment employed. None the less, it represents the base rate in the cohort. One would therefore expect the 945 mothers who described a flu-like illness in the second trimester of pregnancy to yield 945/283.8= 3.33 schizophrenic offspring, if there was no increased risk involved, and $(945 / 283.8) \times 1.87=$ 6.23 , if there was an $87 \%$ increase in risk. The difference between the expected number of 6.23 and the observed number (3) could easily be a chance finding (an exact test gives a probability of 0.15 , or 0.12 if the hypothesis is a $100 \%$ rather than an $87 \%$ increase in risk), confirming our previous claim that Crow \& Done had too few schizophrenic subjects to test their hypothesis adequately. Equally importantly, the figure of 6.23 bears no relationship to the expected figure of $29.5(26.5+3)$ calculated by Crow \& Done.

Exactly similar arguments would apply had narrow diagnostic criteria been used. Indeed, the numbers, both observed and expected, would have been even smaller. We therefore hold to our previous view that Crow \& Done (1992) were not 